\title{
AppliChem
}

\section{maxXbond: first regeneration system for DNA binding silica matrices}

\author{
New solutions allow multiple reuse of valuable material.
}

Silica matrices are a key technology for the purification of DNA. Today the rapid isolation of pure DNA samples is essential for a variety of molecular biology protocols in research and commercial applications. Products with silica matrices are of high quality and high value. Their major disadvantage is that they can only be used once because after elution substantial amounts of DNA remain attached to the silica matrix and the binding capacity is reduced. To solve this problem AppliChem GmbH, Darmstadt in cooperation with multiBIND GmbH, Dortmund developed the first regeneration system for silica matrices. Two innovative solutions remove all nucleic acids and extraneous material from silica matrices and restore the original binding capacity. The regeneration system is commercially available under the name maxXbond. This new product family optimized for the unique regeneration technology allows cost savings of about $70 \%$.

\section{Regeneration technology for silica matrices}

The unique properties of silica matrices for selective DNA binding (Fig. 1a) are the basis for all products related to fast and efficient DNA purification. For more than two decades, more efficient and application-oriented systems for DNA and RNA have been developed ${ }^{1,2}$. Glass powder ('glass milk'; 'batch procedure') or silica columns allow quick and efficient purification procedures ${ }^{3}$. Principles and problems associated with silica matrices are summarized for mini columns (Fig. 1b). Two major tasks for a successful regeneration system are complete removal of residual DNA and restoration of primary binding capacity. Therefore, for a regeneration technology to be useful, the following prerequisites have to be met:

- quick and easy handling

- complete removal of all nucleic acids (both free and trapped)

- no damage to the silica matrix

- complete regeneration of the DNA binding capacity

- affordability.

\section{Karl-Heinz Esser ${ }^{1}$, Wolfram H Marx ${ }^{2}$ \& Thomas Lisowsky ${ }^{1}$}

${ }^{1}$ multiBIND biotec GmbH, Otto Hahn Str. 15, D-44227, Dortmund, Germany. ${ }^{2}$ AppliChem GmbH, Ottoweg 4, D-64291 Darmstadt, Germany. Correspondence should be addressed to T.L. (thomas.lisowsky@gmx.de) or W.H.M. (w.marx@applichem.de).

PUBLISHED ONLINE 20 DECEMBER 2005; DOI:10.1038/NMETH845 a

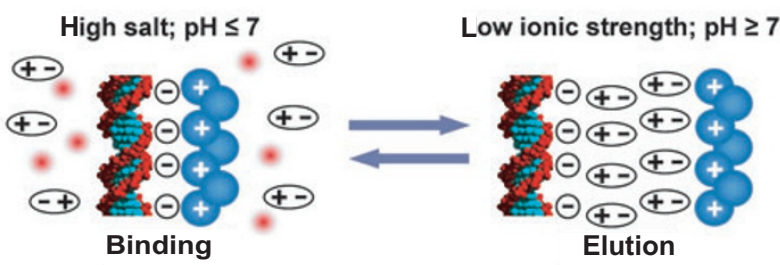

b

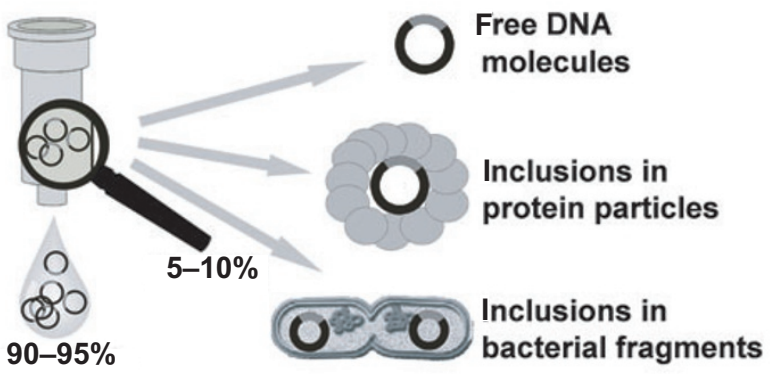

Figure $\mathbf{1}$ | Binding of DNA to silica matrix and mini columns. (a) The purification principle for silica matrices is based on the high affinity of the negatively charged DNA molecule for the positively charged silica particles (blue). Under high salt conditions the DNA is tightly bound and extensive washing removes all contaminations, and purified DNA molecules can be eluted under low ionic strength. (b) With mini-columns, elution removes only $90-95 \%$ of the DNA. The column remains contaminated with DNA molecules and/or inclusions in protein particles or bacterial fragments, and thus has reduced DNA binding capacity.

The two-component maxXbond system with regeneration buffer 1 (RG1) and 2 (RG2) fulfills these requirements. Rapid, efficient regeneration of DNA binding columns takes only 6 min (Fig. 2). Quantitative analysis of DNA yields demonstrates that the binding capacity of regenerated columns is the same as for new columns (Fig. 3a). Analytical agarose gels ${ }^{2}$ and PCR analysis ${ }^{4}$ verify that regenerated columns are nucleic acid-free (Fig. $\mathbf{3 b}$, c). Additional experimental controls for the quality and purity of DNA from regenerated columns are documented in the detailed product information (http://www. applichem.com). A single DNA binding column can be reused at least 20 times $^{5}$.

Additional considerations in the development and success of the new maxXbond regeneration system are the innovative characteristics of the new solutions: 


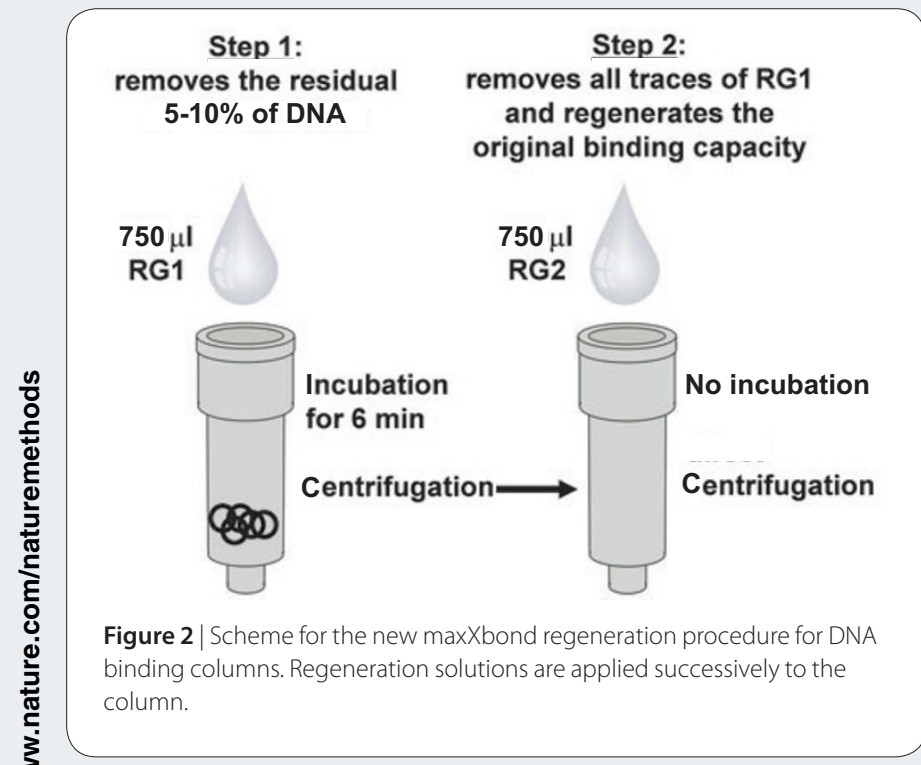

- All components of maxXbond are bio-degradable, harmless or non-toxic for humans.

- No aggressive acids or bases are used. No damage to material or equipment is observed even after prolonged incubation.

- Catalytic and cooperative properties of the maxXbond components cause a rapid and efficient removal or degradation of biological molecules like membrane fragments, proteins and nucleic acids.

- Solutions remain active even in the $\mathrm{pH}$ range from 6 to 8 .

The new maxXbond regeneration system can be applied to all commercially available DNA binding columns that contain silica matrices. Preliminary data indicate that any other DNA binding material like glass powder or minerals can also be regenerated by maxXbond. The new product maxXbond is now available to both academic and industrial scientists who seek to optimize their DNA isolation procedures and save a substantial part of their respective costs. The maxXbond regeneration system and solutions have patents pending.

\section{New product family for efficient regeneration}

The first public presentation of maxXbond at Biotechnica 2005 in Hannover, Germany, generated high interest. The positive feedback of the end users initiated the development of new products optimized for the regeneration cycle. The multiple usage of columns is now supported by a new design of columns and collector tubes.

Increased usage of the regeneration system results in a proportional increase in the demand for standard buffer solutions for DNA purification. This prompted the development of maxXmore: a new universal buffer set for all DNA binding columns with silica matrix. This new five-star buffer system has the following advantages over conventional solutions:

- All five buffers are ready to use.

- No ethanol has to be added to the wash buffer.

- The maxXmore buffer set can be used for all mini columns with silica matrix.

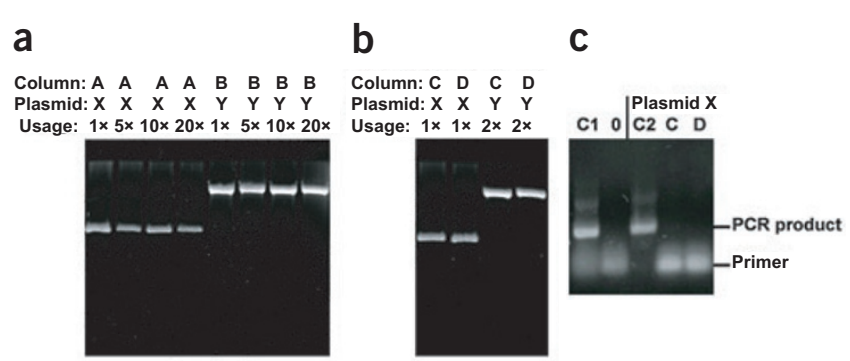

Figure 3 | Quality control of maxXbond-regenerated columns. (a) Restored binding capacity after 20 regeneration cycles. Columns A or B were used and regenerated for 20 cycles of plasmid DNA isolation. Regenerated columns are nucleic acid-free. $(\mathbf{b}, \mathbf{c})$ Columns $C$ and D were used for the isolation of plasmid $X$. After regeneration, plasmid $Y$ was purified with the identical columns; the second DNA isolation of sample $Y$ does not contain any traces of the first DNA sample X (b). PCR analysis does not reveal any DNA molecules from the first isolation (c). Before purification of DNA sample Y, columns $C$ and D were treated with RG1 for $24 \mathrm{~h}$ and 5 min, respectively. Then, $750 \mu \mathrm{l}$ RG2 were applied to each column. Finally, DNA was eluted in $50 \mu \mathrm{l}$ of elution buffer. Then, $2 \mu$ l of eluates were subjected to PCR with appropriate primers for insert in X. C1, control with 1 ng plasmid X DNA; 0 , no DNA; C2, control with $1 \mathrm{ng}$ plasmid $X \mathrm{DNA}$ and $2 \mu \mathrm{l}$ of each eluate after the regeneration of columns $\mathrm{C}$ and $\mathrm{D}$.

- All solutions can be stored at room temperature.

New tests demonstrate that maxXbond also regenerates free silica particles for DNA fragment purification and columns with silica matrix for the purification of PCR products. The latest member of the product family is maxXmore PCR, a buffer set with two optimized solutions for the purification of PCR products with regenerated columns.

\section{Conclusions}

The first regeneration system for silica matrices allows substantial cost savings. The high interest of the scientific community and the positive feedback of the primary users have generated many new ideas for future applications. One can expect that maxXbond may be used with other DNA binding materials and protocols. Presently maxXbond is tested for the use with silica matrices applied to genomic DNA preparations, total RNA isolations or messenger RNA purifications, as well as the regeneration of silica particles, magnetic beads and multi-well plates for automated or high-throughput screening. The optimized version of maxXbond for the complete regeneration of silica-based anion exchange columns will be available soon.

1. Birnboim, H.C. \& Doly, J. A rapid alkaline lysis procedure for screening recombinant plasmid DNA. Nucleic Acids Res. 7, 1513-1522 (1979).

2. Sambrook, J. et al., eds. Molecular Cloning: A Laboratory Manual, 2nd ed. (Cold Spring Harbor Laboratory Press, Cold Spring Harbor, New York; 1989).

3. Vogelstein, B. \& Gillespie, D. Preparative and analytical purification of DNA from agarose. Proc. Natl. Acad. Sci. USA 76, 615-619 (1979).

4. Innis, M.A. et al., eds. PCR Protocols - A guide to methods and applications (Academic Press, Inc., San Diego, 1990).

5. Esser, K. et al. Nucleic acid-free matrix: regeneration of DNA binding columns. Biotechniques 39, 270-271 (2005).

This article was submitted to Nature Methods by a commercial organization and has not been peer reviewed. Nature Methods takes no responsibility for the accuracy or otherwise of the information provided. 